\title{
Depression in the patient with COPD
}

\author{
lan Wilson \\ Department of General Practice, \\ University of Adelaide, SA, Australia
}

\begin{abstract}
This paper explores the recent literature surrounding comorbid depression and COPD. The literature reveals a high prevalence of depression in patients with COPD and some evidence that the depression is a result of the disease. The literature highlights the negative impact of depression on quality of life and a possible impact on mortality. Depression also negatively impacts on compliance and smoking cessation. Treatment of depression in COPD, particularly by cognitive behavioral therapy, has positive impact on quality of life. Tricyclic antidepressants have a positive impact on mood and COPD, but side effects limit their use. The advent of the new antidepressants may improve acceptability and outcomes, but the research is yet to be undertaken. Physical rehabilitation may have a positive impact on mood. This paper highlights the difficulty in screening for depression in patients with COPD due to the overlap of symptoms between the two diseases. Despite the difficulties, it is important to recognize and treat depression in patients with COPD because of the significant likelihood of improvement in quality of life.
\end{abstract}

Keywords: depressive disorder, chronic obstructive pulmonary disease, patient compliance, smoking cessation

\section{Introduction}

Chronic obstructive pulmonary disease is a common chronic lung disease that has a significant impact on the quality of life of those with the condition. It impacts on patients' lives through restriction of activities, interference with sleep, and limitation of social life. It is not surprising that COPD significantly affects the sufferer's mental health. The association between COPD and anxiety and depression is well recognized. This paper explores recent research highlighting the impact of COPD on mood and vice versa. It is based on the author's knowledge of the literature surrrounding the interaction between depression and chronic disease, supplemented by a search of MEDLINE for literature relating to depression or depressive disorder and "pulmonary disease, chronic obstructive".

\section{Prevalence}

There is a well documented association between COPD and anxiety or depression (Dowson et al 2001; Mikkelsen et al 2004; Kunik et al 2005). In respiratory clinics, the prevalence of depression is around 40\% (de Godoy DV and de Godoy RF 2003; Stage et al 2003; Yohannes et al 2003), while in the emergency department and general practice it is lower (12\%, Dahlen and Janson 2002; and 21.6\%, van Manen et al 2002). In general practice, there seems to be a relationship between depression and the severity of the COPD, with $25 \%$ of patients with severe COPD also having depression, $19.6 \%$ of those with mild COPD, and $17.5 \%$ of those in the control group (van Manen et al 2002). In all of the above studies, standard instruments (such as Hamilton and Beck depression scores) were used to make the diagnosis of depression. This is important, as the recognition and diagnosis of depression in nonpsychiatrist practitioners is limited, especially at an isolated consultation 
(Bushnell 2004). The high prevalence of depression in patients attending a respiratory clinic is not unexpected, as the majority of patients will have more severe COPD than that seen regularly in the general practice setting. This argument assumes that the depression is caused by the COPD (see below).

All of the above prevalence rates were determined by diagnostic instruments. Whether screening for depression should be undertaken in patients with COPD also depends on the impact of the disease, its ease of treatment, and the impact of the treatment. As demonstrated below, the recognition and treatment of depression should lead to significant improvements in quality of life.

\section{Impact of depression on COPD}

In patients with COPD seen in the emergency department, the presence of anxiety or depression was associated with significantly higher admission rates $(52 \%$ of patients with anxiety or depression compared with $19 \%$ of those without; Dahlen and Janson 2002). This suggests that depression and anxiety occur in patients with more severe COPD, or that depression and anxiety lead to a worsening of the COPD. In the same study, patients requiring readmission had higher scores on the Hospital Anxiety and Depression (HAD) scale than patients not requiring readmission.

In an outpatient population, the presence of depression or anxiety led to a doubling of the number of days admitted to hospital (Mikkelsen et al 2004). Depression was a better predictor of reduction in activities of daily living than forced expiratory volume in 1 second $\left(\mathrm{FEV}_{1}\right)$ (Yohannes et al 2003). In some studies, depression, among other factors, has been associated with higher mortality (Almagro et al 2002), but this has not been found in all studies (Yohannes et al 2002). Depression in patients with COPD has a significant detrimental impact on quality of life (QOL) (Clary et al 2002). A small study in the elderly demonstrated that the reduction in $\mathrm{FEV}_{1}$ had the biggest impact on QOL, but mood was also important (Peruzza et al 2003).

Depression also has other impacts on patients with COPD. Sleep disturbance is common in COPD, and patients with COPD and depression or arthritis are more likely to have disturbed sleep (Bellia et al 2003). In patients on long-term oxygen therapy, depression was associated with de-creased preference for resuscitation $(50 \%$ not for resuscitation in depressed patients compared with $23 \%$ in nondepressed patients; Stapleton et al 2005). The depression did not have any effect on the preference for mechanical ventilation.

\section{The impact of COPD on depression}

There have been limited studies of the impact of COPD on depression. Pulmonary rehabilitation programs can result in improvement of depression (Kozora et al 2002; Garuti et al 2003; Goldberg et al 2004). Other studies have not demonstrated this (de Godoy DV and de Godoy RF 2003). Whether this is due to improved physical wellbeing, involvement in group activities, or improvement in oxy-genation is unknown.

In the literature on asthma, there is evidence that QOL in patients with asthma and depression is worse than in those patients suffering either alone (Goldney et al 2003). There is also evidence in this literature that symptoms may reflect depression severity rather than asthma severity (Rimington et al 2001). Whether these findings also apply to COPD has not been studied.

\section{Treatment of depression in COPD}

The treatment of depression in patients with COPD has been the subject of a number of reviews (Clary et al 2002; Mikkelsen et al 2004) that have demonstrated the effectiveness of tricyclic antidepressant (TCA) medication in improving mood but with little effect on the COPD. Because of the side effects of TCA there has been a reticence to use them (Clary et al 2002). The advent of selective serotonin reuptake inhibitors (SSRIs) may change this, but so far the studies have been very limited (Clary et al 2002).

Several reviews of cognitive behavioral therapy (CBT) (Clary et al 2002; de Godoy DV and de Godoy RF 2003; Mikkelsen et al 2004) have demonstrated its effectiveness in improving physical as well as psychological wellbeing. These reviews have supported the use of CBT as an adjunct to pulmonary rehabilitation.

The positive impact of improving mood in response to rehabilitation suggests that the diminished mood may be secondary to COPD. A difficulty arises in that at least some of the symptoms of depression, as measured in depression rating instruments, may arise from the COPD itself. Tiredness, lack of energy, weight loss, and disturbed sleep are symptoms of both conditions.

The diminished cognition and mental slowing may be attributable to hypoxia (Clary et al 2002; Mikkelsen et al 2004), and depression itself may be secondary to corticosteroid administration (Mikkelsen et al 2004). Treatment of COPD may improve the hypoxia and lead to a reduction in corticosteroid administration. The association 
of mood with corticosteroid administration is complex, with several possible outcomes. Clinical experience suggests that a significant number of people taking corticosteroids have an improvement in their mood, yet drug-induced psychosis is also seen. People who are on reducing doses of corticosteroid medication may develop tiredness and joint pains. The impact of corticosteroids in the individual patient is idiosyncratic.

\section{Cause and effect}

There have been few longitudinal studies of the development of depression in patients with COPD. The major study, the Health and Retirement Study (Polsky et al 2005), followed 8387 adults aged 51-61 years for 10 years. Subjects were reevaluated biennially. Part of this study examined the occurrence of depressive symptoms after the diagnosis of one of seven medical conditions. Chronic lung disease (excluding asthma) was included in the analysis. Within two years of diagnosis the hazard ratio for the development of depressive symptoms was 2.21 (95\% confidence interval 1.64-2.97). This compares with 3.55 for cancer and 1.45 for heart disease. Depressive symptoms developed 2-4 years after the diagnosis of inflammatory arthritis. The Health and Retirement Study supports the idea that depression in COPD develops in response to the diagnosis of the disease.

\section{COPD, depression, and smoking}

A number of studies have demonstrated that patients with COPD who also suffer from depression or anxiety are more likely to be smokers (van Manen et al 2002; Wagena et al 2004). The primary association appears to be between depression and smoking (Almeida and Pfaff 2005; Gulec et al 2005). Anecdotally, psychiatrists report that cigarette smoking appears to have an antidepressant effect. Generally, depression severely limits the effectiveness of smoking cessation programs (Cinciripini et al 2003; Freedland et al 2005).

\section{Depression and compliance}

On theoretical grounds, it has been assumed that depression is associated with poor compliance. This has been confirmed in studies that relate to asthma treatment. In a prospective study, poor compliance (taking less than $70 \%$ of inhaled medication) was associated with higher depression scores at commencement of the study (Bosley et al 1995). Although anxiety levels were also high, anxiety was not associated with poor compliance. It is possible to postulate that anxiety actually improves compliance, by focusing the patient on their disease and its treatment. Other cross-sectional studies have confirmed the findings of poor compliance being associated with higher levels of depression (Cluley and Cochrane 2001).

\section{Screening for depression}

Most of the studies described previously have used diagnostic instruments to diagnose depression. A few have used the "gold standard" of psychiatric interview. There have not been any studies of screening instruments.

In the general literature on depression there have been some attempts at developing screening tools, but these have not always been successful. The most commonly examined screening instruments are the one and two question screens (Whooley et al 1997; Arroll et al 2003; Henkel et al 2004). These studies demonstrate that the two questions, which relate to the two essential criteria for the diagnosis of depression, are effective. However, preliminary work (Reddy et al 2005) indicates that such screening tests are ineffective in patients with chronic diseases. Tiredness, low energy, weight loss, and loss of interest occur in both depression and COPD.

It would seem that instruments such as the Beck Depression Inventory or the Hamilton Depression scale are required. Unfortunately, these require some time for the patient to complete and the practitioner to interpret. Using them in busy clinical practice as screening instruments is not practical. It would seem appropriate that screening tests for depression in patients with COPD still need to be devised.

\section{Conclusions}

Depression is common in patients with COPD, and its presence has a significant impact on the QOL of such patients and may be associated with a higher mortality rate. It also negatively affects compliance and smoking cessation.

Depression responds to treatment and the literature suggests that cognitive behavioral therapy may be the treatment of choice. Further research is required to determine the impact of SSRIs in the treatment of depression in patients with COPD and the cost benefit of screening for depression in patients in different settings. Care needs to be taken in developing screening tests for depression in patients with COPD. 


\section{References}

Almagro P, Calbo E, Ochoa de Echaguen A, et al. 2002. Mortality after hospitalization for COPD. Chest, 121:1441-8.

Almeida OP, Pfaff JJ. 2005. Depression and smoking amongst older general practice patients. J Affect Disord, 86:317-21.

Arroll B, Khin N, Kerse N. 2003. Screening for depression in primary care with two verbally asked questions: cross sectional study. BMJ, $327: 1144-6$.

Bellia V, Catalano F, Scichilone N, et al. 2003. Sleep disorders in the elderly with and without chronic airflow obstruction: the SARA study. Sleep, 26:318-23.

Bosley CM, Fosbury JA, Cochrane GM. 1995. The psychological factors associated with poor compliance with treatment in asthma. Eur Respir J, 8:899-904.

Bushnell J. 2004. Frequency of consultations and general practitioner recognition of psychological symptoms. Br J Gen Pract, 54:838-43.

Cinciripini PM, Wetter DW, Fouladi RT, et al. 2003. The effects of depressed mood on smoking cessation: mediation by postcessation self-efficacy. J Consult Clin Psychol, 71:292-301.

Clary GL, Palmer SM, Doraiswamy PM. 2002. Mood disorders and chronic obstructive pulmonary disease: current research and future needs. Curr Psychiatry Rep, 4:213-21.

Cluley S, Cochrane GM. 2001. Psychological disorder in asthma is associated with poor control and poor adherence to inhaled steroids. Respir Med, 95:37-9.

de Godoy DV, de Godoy RF. 2003. A randomized controlled trial of the effect of psychotherapy on anxiety and depression in chronic obstructive pulmonary disease. Arch Phys Med Rehabil, 84:1154-7.

Dowson C, Laing R, Barraclough R, et al. 2001. The use of the Hospital Anxiety and Depression Scale (HADS) in patients with chronic obstructive pulmonary disease: a pilot study. N Z Med J, 114:447-9.

Freedland KE, Carney RM, Skala JA. 2005. Depression and smoking in coronary heart disease. Psychosom Med, 67:S42-6.

Goldney RD, Ruffin R, Fisher LJ, et al. 2003. Asthma symptoms associated with depression and lower quality of life: a population survey. Med $J$ Aust, 178:437-41.

Gulec M, Bakir B, Ozer M, et al. 2005. Association between cigarette smoking and depressive symptoms among military medical students in Turkey. Psychiatry Res, 134:281-6.
Henkel V, Mergl R, Coyne JC, et al. 2004. Screening for depression in primary care: will one or two items suffice? Eur Arch Psychiatry Clin Neurosci, 254:215-23.

Kunik ME, Roundy K, Veazey C, et al. 2005. Surprisingly high prevalence of anxiety and depression in chronic breathing disorders. Chest, 127:1205-11.

Mikkelsen RL, Middelboe T, Pisinger C, et al. 2004. Anxiety and depression in patients with chronic obstructive pulmonary disease (COPD). A review. Nord J Psychiatry, 58:65-70.

Peruzza S, Sergi G, Vianello A, et al. 2003. Chronic obstructive pulmonary disease (COPD) in elderly subjects: impact on functional status and quality of life. Respir Med, 97:612-17.

Polsky D, Doshi JA, Marcus S, et al. 2005. Long-term risk for depressive symptoms after a medical diagnosis. Arch Intern Med, 165:1260-6.

Reddy P, Bunker S, Dunbar J. 2005. In: General Practice \& Primary Health Care Research Conference: Getting research right for policy and practice. Primary Health Care Research and Information Service; 2005 Jul 26-28; Adelaide, SA, Australia. p 148.

Rimington LD, Davies DH, Lowe D, et al. 2001. Relationship between anxiety, depression, and morbidity in adult asthma patients. Thorax, 56:266-71.

Stage KB, Middelboe T, Pisinger C. 2003. Measurement of depression in patients with chronic obstructive pulmonary disease (COPD). Nord $J$ Psychiatry, 57:297-301.

van Manen JG, Bindels PJ, Dekker FW, et al. 2002. Risk of depression in patients with chronic obstructive pulmonary disease and its determinants. Thorax, 57:412-16.

Wagena EJ, Kant I, Huibers MJ, et al. 2004. Psychological distress and depressed mood in employees with asthma, chronic bronchitis or emphysema: a population-based observational study on prevalence and the relationship with smoking cigarettes. Eur J Epidemiol, 19:147-53.

Whooley MA, Avins AL, Miranda J, et al. 1997. Case-finding instruments for depression. Two questions are as good as many. J Gen Intern Med, 12:439-45.

Yohannes AM, Baldwin RC, Connolly M. 2002. Mortality predictors in disabling chronic obstructive pulmonary disease in old age. Age Ageing, 31:137-40.

Yohannes AM, Baldwin RC, Connolly MJ. 2003. Prevalence of subthreshold depression in elderly patients with chronic obstructive pulmonary disease. Int J Geriatr Psychiatry, 18:412-16. 